\title{
Based on the comparison of Chinese and foreign teaching methods of Higher Mathematics in Higher Vocational Colleges ---- The concept of the derivative as an example
}

\author{
Haiyan Zhen \\ Shandong Institute of Commerce and Technology Jinan, 250103, China \\ email:13764660@qq.com
}

Keywords: Higher Mathematics; Higher Vocational Colleges;Theory of nature; Teaching Methods

\begin{abstract}
As an important public basic course in higher vocational colleges, higher mathematics is not only the foundation of learning other specialized course, but also it is an important carrier of the training of students' comprehensive quality. However, about the teaching of higher mathematics in higher vocational colleges in our country, the teaching arrangement and teaching methods can't pay attention to the cultivation of students' ability to solve practical problems. The majority of students believe that mathematics is useless so that they are not interested in the studying of higher mathematics. Combined with the teaching book Calculus: Early Transcendental (5thed) of foreign high-quality vocational education resources, About the concept of derivative, for example, we carry on comparative analysis to higher mathematics teaching of Chinese and foreign higher vocational colleges, then put forward an important enlightenments on the higher mathematics teaching in higher vocational colleges in our country.
\end{abstract}

Higher mathematics as a basic public required course in higher vocational colleges, on the one hand, it is the important foundation of students' learning related professional knowledge; on the other hand it plays an important role in improving students' comprehensive quality and cultivating students' learning ability. Higher vocational education in China started relatively late, higher mathematics teaching in higher vocational education mainly follows the teaching methods of ordinary colleges and universities. Textbooks generally are concise editions of Higher Mathematics teaching materials of ordinary colleges and universities. The arrangement of teaching material and teacher's teaching reflects the characteristics of heavy theory, light practice. At the same time, not pay attention to train the students' ability of applying mathematical knowledge to solve practical problems, thus easy for students to produce the concept of "mathematics is useless", and can't arouse the students' interest in learning higher mathematics. A survey found that the majority of our students do not know how to make full use of the knowledge of mathematical to solve practical problems after school, even if it is a very simple math problem, they would ask for the help of professionals. The reason is mainly because of lack of some good training during the school, along with lack of the awareness of the applying their own learning mathematical knowledge at the university to the practical problems life. Abroad Higher Mathematics Teaching in terms of the arrangement of the materials or the method of teaching is more attentive to mathematics awareness of students, and relations between mathematics and everyday life. In the teaching and practice, for example, choose some things students are familiar with. Focus on the cultivation of the students' ability to use the knowledge of advanced mathematics to solve practical problems. Combined with the teaching book for foreign high-quality vocational education resources ,Calculus: Early Transcendental(5thed), which is written by James Stewart ,Bai Fengshan mainly translated. Teaching of the concept of the derivative as an example, higher mathematics teaching methods of Chinese and foreign vocational colleges were analyzed and propose a significant enlightenment to Higher Vocational Mathematics Teaching in China.

Derivative is the foundation of calculus, and an important tool of study the function to solve practical problems. The definition of the concept of the derivative in textbooks of higher vocational college in China is similar. First is the case, slope of the tangent and speed of non-uniform motion 
are usually used, and then directly cut into the theme of explaining the concept of the derivative. Emergence of the cases is very stiff, and fewer teachers explain the relationship of the cited cases with derivative, even a beginner often does not understand cited cases appear here. After the introduction of the concept of derivative is the derivative calculation method, the whole process is boring, does not reflect the value of the application of mathematics. This way of teaching is not conducive to the cultivation of students' consciousness about the application of math, and students cannot establish a contact between math with the issues in real life .What is worse, in the future work and life it is very difficult for them to be able to think of the derivative as a good method to solve some practical problems. For that, on Calculus: Early Transcendental (5thed) written by James Stewart, the definition of the derivative has a good reference. James Stewart first lists lots of examples, which were involved in various fields, to fully reveal the nature of derivatives, so that readers can fully grasp the essence of derivative and its application value. On the basis of the above, draws the concept of derivative, then go back and then further exploit the meaning of derivative to solve practical problems in all areas of life. The process of James Stewart arranging the concept of derivative is introduced is as follows: firstly, the introduction of instances:

(1)Tangent: Suppose the equation of the curve $C$ is $y=f(x)$, find the tangent of $C$ at the point $P(a, f(a))$, first find a point $Q(x, f(x)), x \neq a$ in the vicinity of the point $\mathrm{P}$, calculate the slope $m_{P Q}=\frac{f(x)-f(a)}{x-a}$ of the secant $P Q$, When $\mathrm{x}$ approaches a, namely the point $\mathrm{Q}$ approaches the point $\mathrm{P}$, If $m_{P Q}$ approaches a constant $\mathrm{m}$, then the straight line $\mathrm{m}$ passing through the point $\mathrm{P}$ is a tangent.

(2)Speed: Suppose equation of motion of the object along the linear motion is $s=f(t)$, the average speed from $t_{1}$ to $t_{2}$ is $\frac{f\left(t_{2}\right)-f\left(t_{1}\right)}{t_{2}-t_{1}}$, when $t_{1}$ Approaching $t_{2}$, average speed is the instantaneous speed of the object in time $t_{1}$. That is $v\left(t_{1}\right)=\lim _{t_{2} \rightarrow t_{1}} \frac{f\left(t_{2}\right)-f\left(t_{1}\right)}{t_{2}-t_{1}}$.

Cited cases for the concept of the derivative in our country's teaching material will stop here, then the concept of the derivative should be the introduced, generally later the relationship between the derivative and other practical problems will not be mentioned, and instead of putting forward the concept of the derivative, James Stewart continues to introduce instances so as to further guide the students to fully reveal the nature of the derivative. At the same time, continue to introduce examples from physics, chemistry, biology and psychology.

(1)Application in the field of physics: The instantaneous change rate of mass (linear density of the non-uniform $\operatorname{rod} \rho$ ), the instantaneous change rate of the power through the wire (instantaneous current intensity $I$ ), the instantaneous rate of change of the temperature of the object (instantaneous cooling rate $T$ ), and the like.

Linear density of the non-uniform rod: Has a non-uniform thin rods, take the end of bar as the origin, the distribution for the coordinates of any point on the rod is in the interval $[0, x]$, the mass $\mathrm{m}$ of stick is a function $m=f(x)$ of $\mathrm{x}$, The mass of the rod lies between $x=x_{0}$ and $x=x$ is $f(x)=f\left(x_{0}\right)$, average density of this part of rod is $\frac{f(x)-f\left(x_{0}\right)}{x-x_{0}}$ whose limit is rate of change of the mass of the rod under $x_{0}$ with respect to the length, when $x \rightarrow x_{0}$, that is the linear density of the rod at the point $x_{0}$ namely $\rho\left(x_{0}\right)=\lim _{x \rightarrow x_{0}} \frac{f(x)-f\left(x_{0}\right)}{x-x_{0}}$.

Instantaneous current intensity: Suppose current through the wire is non-steady, at time t, power through cross section of wire is $Q=f(t)$, from $t_{0}$ to $t$ the current through cross section of wire is $Q=f(t)-f\left(t_{0}\right)$. Average current through it in this period is $\frac{f(t)-f\left(t_{0}\right)}{t-t_{0}}$, when 
$t \rightarrow t_{0}$, the limit is rate of change of conductor of electricity at time $t_{0}$ with respect to time, namely the instantaneous current through the wire in time $t_{0}$. That is $I\left(t_{0}\right)=\lim _{t \rightarrow t_{0}} \frac{f(t)-f\left(t_{0}\right)}{t-t_{0}}$.

Cooling rate: When the temperature of the object is higher than the temperature of the surrounding medium, the object will continue to cool. If the functional relationship between the temperature of object $T$ and time $t$ is $T=f(t)$. From a certain moment $t_{0}$ to the time $t$, average temperature change of the object is $\frac{f(t)-f\left(t_{0}\right)}{t-t_{0}}$, when $t \rightarrow t_{0}$, the limit is change rate of the temperature of the object at time $t_{0}$ with respect to time, namely $T\left(t_{0}\right)=\lim _{t \rightarrow t_{0}} \frac{f(t)-f\left(t_{0}\right)}{t-t_{0}}$.

(2) Application in the field of chemistry: The reaction rate. Set up a chemical reaction equation: $A+B=C$, The concentration $N$ of the resultant $C$ is a function of time $t$, that is $N=f(t)$.so from a certain time $t_{0}$ to time $t$, the average reaction rate of $C$ is $\frac{f(t)-f\left(t_{0}\right)}{t-t_{0}}$ whose limit is instantaneous reaction rate of the resultant $C$ at time $t_{0}$, when $t \rightarrow t_{0}$. Namely $N\left(t_{0}\right)=\lim _{t \rightarrow t_{0}} \frac{f(t)-f\left(t_{0}\right)}{t-t_{0}}$.

(3)Applications in biology: The instantaneous rate of growth of the bacteria. Changes in the number of bacteria is a discontinuous function, but for some cases containing a particularly large number of bacteria, we can assume that a certain number $S$ of bacteria is a continuous function of time $t: S=f(t)$. From a certain time $t_{0}$ to time $t$, the average rate of change of the number of bacteria is $\frac{f(t)-f\left(t_{0}\right)}{t-t_{0}}$. When $t \rightarrow t_{0}$, the limit of $\frac{f(t)-f\left(t_{0}\right)}{t-t_{0}}$ is instantaneous rate of change of the number of bacteria $C$ at time $t_{0}$.Namely $S\left(t_{0}\right)=\lim _{t \rightarrow t_{0}} \frac{f(t)-f\left(t_{0}\right)}{t-t_{0}}$.

(4)Application in the field of psychology: The rate of change in academic performance. Assume that the result $C$ of learning a subject is a function $C=f(t)$ of learning time $t$. From a certain time $t_{0}$ to time $t$, the average rate of change of the result is $\frac{f(t)-f\left(t_{0}\right)}{t-t_{0}}$. When $t \rightarrow t_{0}$, the limit is an instantaneous rate of change of the result $C$ at time $t_{0}$. Namely $C\left(t_{0}\right)=\lim _{t \rightarrow t_{0}} \frac{f(t)-f\left(t_{0}\right)}{t-t_{0}}$.

James Stewart, through the introduction of a large number of examples, makes the readers fully understand what they have in common, and then the definition of derivative is followed. Leading to the definition of the derivative is not the end, and further reveals that for the reader: the performances of derivative in various fields are different, but the essence is the same. Namely, the rate of change is the most essential meaning of the derivative. It brings out the crucial point which is conducive for students to understand of the applications of derivatives. And he gives a large number of exercises which is about making use of the essential meaning of derivative to solve practical problems, for example: when cars are driven with a speed of $v$ mile/h. Its fuel consumption is $C=f(v)$, Q (1) what's the meaning of derivative $f^{\prime}(v)$ ? What is the unit? (2) Explain the meaning of $f^{\prime}(20)=-0.05$ in a simple language. Another good example: in the lab, after thours, the number of the bacteria is $n=f(t)$, Q (1) what's the meaning of derivative $f^{\prime}(5)$ ?, and what is the unit of the derivative? Q (2) Given the bacteria is provided a unlimited number of space and a unlimited number of space nutrients, do you think which is better between $f^{\prime}(5)$ and $f^{\prime}(10)$ ? If the number of the nutrient is limit, it will affect your conclusion? Please explain the above question. 
A lot of practice further strengthen the derivative value of practical application; also make the students have a further and full understanding on the essential meaning of derivative. This way of teaching deepen students' understanding of mathematical knowledge, conducive to develop their mathematical problem view and the consciousness of constructing mathematical model, helping train the ability of the students in vocational colleges to use mathematical methods to solve practical problems. Thus it is easier for graduate students to apply mathematics to practical work.

The potential for the application of mathematics lies in its theoretical nature, only have a thorough understanding of the nature of the derivative, can we make it better applied to a variety of fields. About nature characteristics of Mathematical, the famous French mathematician Joseph Fourier summarized it: Math make a lot of different phenomena together compared and reveal similarities between them [1]. James Stewart's teaching in the higher mathematics embodies the thought of Joseph Fourier, but the higher vocational mathematics education in China is still just from the knowledge structure of mathematical, focus on the "teach" ignore "discovery", result in a highbrow situation so many students have an impression that the learning of higher mathematics is useless and difficult. From the needs of studying and life, and to make an overall considerations of lifelong learning and development for students in vocational colleges, and they can make better and full use of the knowledge of mathematics to solve practical problems in many areas, as the educators, we should continue to explore, and to find more teaching methods stimulating students' thinking, in order to improve their learning interest and the awareness of the studying, so the teaching method of James Stewart has important implications for teaching of higher mathematic in China.

\section{References}

[1] Beijing Higher Education Publication: Bai Fengshan as main translation, Calculus (Volume 1), pp. 157-171,2004[James Stewart, Calculus:Early Transcendentals(5th ed) US].

[2] Yujuan Jiang, Wang Lili, "Analysis of the present situation and a first exploration on reformation of higher mathematics teaching in Higher Vocational Colleges,"academic journal of Harbin Vocational and Technical College, vol. 5, 2009.

[3] Suyun Zhao, Zhao Zhichuan, "Comparison and reflection on Chinese and foreign quality education,” Chinese Adult Education, vol. 5, 2006. 